\title{
Four-Dimensional Imaging: Computer Visualization of 3D Movements in Living Specimens
}

\author{
C. Thomas, ${ }^{*}$ P. DeVries, J. Hardin, J. White
}

The study of many biological processes requires the analysis of three-dimensional (3D) structures that change over time. Optical sectioning techniques can provide 3D data from living specimens; however, when $3 \mathrm{D}$ data are collected over a period of time, the quantity of image information produced leads to difficulties in interpretation. A computer-based system is described that permits the analysis and archiving of 3D image data taken over time. The system allows a user to roam through the full range of time points and focal planes in the data set. The user can animate images as an aid to visualization and can append multicolored labels and text notes to identified structures during data analysis. The system provides a valuable tool for the study of embryogenesis and cytoplasmic movements within cells and has considerable potential as an educational tool.

Living things, by their very nature, are dynamic. Individual cells undergo complex rearrangements of their cytoarchitecture as they progress through the cell cycle or become terminally differentiated. Groups of cells undergo coordinated shape changes and migrations during the development of shape and form in an embryo. Understanding these processes is a fundamental goal of the developmental or cell biologist. Traditionally, biologists have studied these phenomena by fixing the specimen at a defined time point, embedding it in a plastic or wax matrix, and cutting sections for observation under the microscope. By repeating this process at differing time points, it is possible to infer dynamic behavior, provided the hehavior being studied is sufficiently stereotyped to enable reconstruction from discrete time points taken from different specimens. However, even in these cases, the inference of continuous behavior from sparsely sampled discrete time points can lead to misinterpretation, particularly if the processes being studied have intrinsic variability.

Optical sectioning microscopy has become a very powerful tool for the biologist because it allows a single focal plane to be observed within an intact specimen with minimal image degradation caused by outof-focus interference. Optical sectioning is noninvasive and is therefore well suited to the study of living specimens. It allows the 3D dynamic behavior of a given organism to be directly and unambiguously inferred.

C. Thomas, P. DeVries, and J. White are with the Integrated Microscopy Resource for Biomedical Research (IMR), University of Wisconsin, Madison, WI 53706, USA. $\mathrm{J}$. Hardin is in the Department of Zoology, University of Wisconsin, Madison, WI 53706, USA.

*To whom correspondence should be addressed.
The first optical sectioning technique to be developed was differential interference contrast (Nomarski) imaging. The technique contrasts the spatial rate of change of refractive index within a specimen. It provides good optical sectioning capabilities near the surface of a specimen, although light scattering can degrade images from deep sections. Nomarski imaging gives good visualization of certain cellular organelles, but it lacks the specificity needed for molecular identification.

Specific visualization of a molecular species within a sample is often accomplished by introducing into the specimen a ligand, such as an antibody, that specifically binds to a target entity under investigation. The ligand is usually attached to a fluorescent probe (either directly or indirectly) and visualized by fluorescence microscopy. Alternatively, the gene for a naturally fluorescent protein may be appended to the gene coding for the protein under study or its promoter (1). When an organism is genetically transformed with this construct, the cells expressing the protein under study can be visualized by in vivo fluorescence.

Recently a number of techniques have become available for optical sectioning fluorescence microscopy, such as confocal imaging (2), multiple-photon excitation imaging (3), and computational deconvolution of wide-field images (4). Fluorescence techniques are more difficult to apply to living specimens than is bright-field microscopy because of the possibility of damaging specimens by phototoxicity. Nevertheless, great progress has been made in this area in recent years, and many new biological insights have arisen through the use of in vivo optical sectioning fluorescence imaging.

The various optical sectioning microsco- pies that are available can provide the necessary raw information to reconstruct dynamic events in three dimensions by collecting stacks of images from through-focal series at regular time intervals (5). The vast amount of data that can be generated makes such a task daunting. For example, in our studies of cell lineage in the developing embryo of the nematode Caenorhabditis elegans, we have empirically determined that we need to take a minimum of 20 optical sections over 1000 time points $(20,000 \mathrm{im}$ ages) to be able to follow all the embryonic cell divisions. Moreover, if the time points are sufficiently close to allow events to be reconstructed accurately, the differences between time points will often be small and difficult to perceive.

The eye and brain are particularly adept at detecting movement in an image if the movement occurs reasonably rapidly. However, if the changes occur slowly, it is very difficult to discern the nature of these changes. One cannot see how grass grows, for example. If a time-lapse movie is made so that the movements are speeded up to a value that is optimal for perception, then the process of growth can be readily observed. Imposed movements can help to visualize static structures. For example, a microscopist typically moves the focus of a microscope when observing a specimen instead of observing static planes of focus; movements that are detected as the focus is adjusted give an impression of how specimen shape changes in the axial direction. In this way, the perception of movement is used to convey 3D information.

We describe a system that can record and archive time-sequential stacks of optical sections into a four-dimensional data set (a 4D image archival system). The system then allows the user to roam around this data set to reconstruct the dynamic $3 \mathrm{D}$ events that occurred during data collection. A user can roam forward and backward in time and can animate the images for easier perception of changes. Alternatively, or in addition, a user can roam up and down in focus, either to track a moving object in three dimensions or to convey through animation how a structure changes shape axially.

\section{Design Considerations for a 4D Image Archival System}

The vast amounts of information in a $4 \mathrm{D}$ data set can be visualized in several ways. Volume rendering techniques can be used to represent the entire 3D volume for each time point (6). In addition to being very computationally intensive, volume rendering techniques have several disadvantages. 
When the information for each time point is viewed as a "solid" 3D object, there are invariably parts of the data set that are obscured by objects in the foreground. The obscured structures are usually in the interior of the volume or in the portions of the sample that are facing away from the viewer. Additionally, some of the features of 3D rendering that make it an attractive method of display (such as lighting models, surface texturing, and adjustable opacity) force the rendering algorithms to make decisions about the location and visibility of surfaces within the data set. An alternative way to deal with 4D data is to display the raw data, as collected, two dimensions at a time. This method is simple; no computationally intensive algorithms are needed. Instead, the 2D images are displayed as an animation, so that the formidable feature recognition capabilities of the viewer's eye and brain can be put to effective use.

A major design goal of the 4D imaging project was to create a system that would be able to economically store the 20,000 or more images needed to reconstruct a $C$. elegans lineage. In addition, the playback roam around a 4D data set quickly enough to be able to use animation as a visualization aid. Early versions of the system enabled us to follow embryonic lineages, but it became apparent that a method of applying labels to identified objects (for example, particular cells in an embryo) was desirable. We also found it useful to associate descriptive notes with any particular label. In developing a system of labeling, we felt it was important that the labels could be edited at any time, that the display of the labels could be toggled on and off by the user, and that component had to provide the ability to

the original image data should not be altered in any way.

Four-dimensional archiving of images of embryonic development can yield a vast amount of data. For example, C. elegans embryonic development takes place over an 8 -hour period. The embryo is $\sim 50 \mu \mathrm{m}$ in the long axis and $25 \mu \mathrm{m}$ across. The lateral resolution limit of a light microscope is typically $250 \mathrm{~nm}$. Oversampling by a factor of 2 , so that quantization errors minimally degrade resolution, requires a minimum image of 400 by 200 pixels. The axial resolution of a microscope is typically about onethird to one-half as good as its lateral resolution; therefore, about 50 optical sections would be needed. A pixel depth of 6 to 8 bits would yield contrast discrimination at least as good as that of the eye. A time interval of $30 \mathrm{~s}$ between images would be sufficient to capture the fastest events in C. elegans development; for the 8-hour period, images at $\sim 1000$ time points would thus be required. The total file size for a $4 \mathrm{D}$ data set of $\mathrm{C}$. elegans development would therefore be $\sim 4$ gigabytes $(50,000$ images, each of 80 kilobytes).

Although this amount of data is inconveniently large, it can be managed in several ways. Analog optical disk recorders are capable of storing $\geq 20,000$ video frames on a single surface of the recording medium; computer control enables random access to any given frame or sequence of frames. If the data are organized as a set of contiguous frames within each focus level, then these devices are capable of rapid animation when roaming backward and forward in time at a fixed focus level. Four-dimensional archiving systems have been developed using these devices $(7,8)$

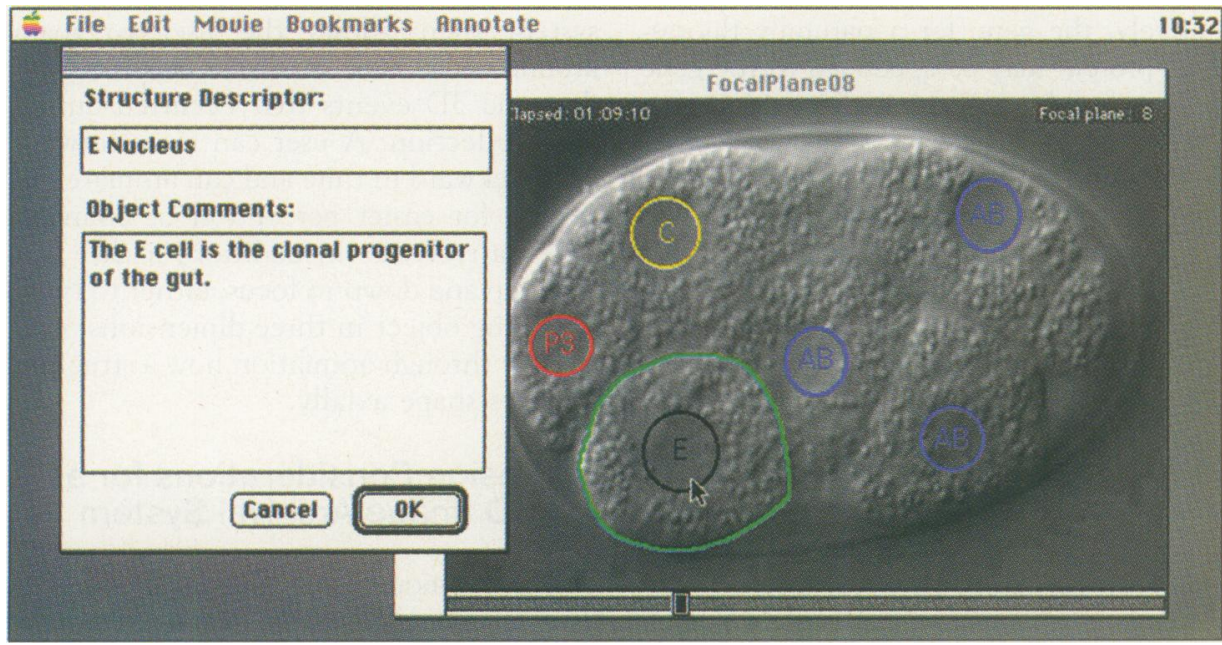

Fig. 1. A Nomarski image of a single focal plane from the interior of a $C$. elegans embryo in the early part of its developmental sequence, as viewed by the 4D Viewer application. Nuclei visible in this focal plane have been highlighted with color-coded labels; the E cell (Fig. 5) is outlined in green. In addition to creating dynamic color overlays, the 4D Viewer can also associate hidden text with each overlay object; here, annotation is displayed in a dialog box by clicking the mouse on the item in question. and can be valuable tools for determining cell lineages (9) and characterizing cytoplasmic movements within cells (7). However, analog optical disk recorders suffer from two disadvantages when used in this application: the data storage medium is expensive and often not rewritable, and it is difficult to attach labels or overlays to the data during analysis without corrupting the original data.

Data compression techniques can reduce the data storage requirements of digitally coded image data. Compression techniques make use of the inherent redundancy within an image to reduce the amount of data that encodes the image. There are two general strategies: lossless compression that maintains all the original information within the image, and lossy compression schemes that allow trade-offs in data reduction and image fidelity. We have found that a lossless scheme typically yields a factor of $\sim 2$ data reduction. However, lossy schemes such as JPEG compression (an algorithm defined as an international standard by the Joint Photographic Experts Group) can give data compressions of more than a factor of 15 while maintaining visually acceptable image quality. Within the JPEG algorithm, the degree of compression can be tailored for each data set; high compression can be used with a highly redundant data set without sacrificing much image fidelity, whereas low compression must be used with a data set that has less redundancy if preservation of image fidelity is a concern. The JPEG algorithm is commonly used to encode images for transmission across the Internet, and the software for encoding and decoding is readily available and is often incorporated into the operating systems of desktop computers. Encoding and decoding images with the JPEG algorithm is computationally intense; however, the current generation of processors used in desktop computers can decode a sequence of JPEG-encoded images quickly enough to provide animation. We find that a Nomarski image can be compressed with the JPEG algorithm up to a factor of 20 . Compression by a factor of 10 to 15 generally maintains excellent fidelity of a $\mathrm{No-}$ marski image (Fig. 1) and is the value that we routinely use.

\section{Integrated Microscopy Resource 4D Image Archival System}

The 4D system described here was a further development of an earlier system that was based on analog video disks (7) and an alldigital system (10). The system was implemented on relatively inexpensive desktop computers (Apple Macintosh PowerPCs) 
and uses no purpose-built hardware (11). The system has three components: the 4D Acquisition software, the 4D Turnaround program, and the 4D Viewer program.

Data acquisition system. The 4D Acquisition software system for video microscopy, an NIH Image-based recording system with data acquisition macros (12), allows the user to gather multiple $3 \mathrm{D}$ data sets at various time points throughout the duration of observation. The software coordinates the function of the frame-grabber card, the microscope stage drive motor, and an optional illumination shutter (Fig. 2). The hardware components are controlled to allow either the imaging of a single focal plane of a specimen over time or the sampling of 3D volumes into multiple focal slices at each time point.

After the sample is mounted and the microscope imaging conditions are optimized such that the items of interest in the sample are easily visualized, live capture from the microscope video camera is enabled and the image from the microscope is displayed on the computer screen. The region of interest (ROI) is selected in the camera window by using the mouse to drag a rectangle around it. After ROI selection, the 4D Acquisition software is run. The software prompts the user to specify the number of time points to be acquired and to select either a uniform time delay (for example, sampling every $30 \mathrm{~s}$ ) or a variable time delay between acquisitions. Because organisms generally do not develop at a constant rate, the software allows the user to specify a set of collection intervals that will be used during the course of an experiment to match the rate of development of the specimen under observation. The variable collection rate feature further reduces the image data storage requirements by sampling less frequently at times when development proceeds more slowly.

After deciding between static and variable time delays for image acquisition, the user chooses between collecting data from a single focal plane and collecting a 3D data set of slices at each time point. A user who elects to sample three dimensions at each time point is prompted to select the top and bottom focal planes of the sample by means of a graphic interface to the microscope focus control. After moving through the sample and marking the top and bottom, the user then specifies either the number of focal planes to be acquired or the increment for the focus motor to move between focal planes (given one parameter, the software will determine the other). Finally, the user is prompted for a file name, for a location on the hard drive to store the images, and for an image file format to use when saving the images. Currently, the software supports
TIFF, PICT, and PICS file formats. The images are initially stored uncompressed (to allow access to the unaltered data if quantization is required) but can be compressed later for archiving.

When a recording run begins, the software automatically moves the microscope focus through the specified volume, digitally sampling and storing images of each focal plane. Each time-point file contains a stack of images representing the 3D structure of the sample (Fig. 3). During the interval between time points, the illumination shutter closes to block unnecessary light from the specimen and the time-point file is written to the computer disk.

The 4D Acquisition system can be used to record data from any optical sectioning microscope that can provide a video output.

For Nomarski microscopy, we reduce any residual noise on the signal by means of frame averaging (13). We have found that cleaning up the signal in this way allows the digital image to be compressed up to an additional $25 \%$ while maintaining equivalent image fidelity. For confocal or multiple-photon images, we use simple macros (written in Bio-Rad 600 SOM software) to collect 4D data sets.

Data compression and turnaround. It is not unusual to generate several thousand images, occupying a gigabyte or more of hard disk space, from a single 4D data acquisition session. This amount of data is too unwieldy for the personal computer technology currently available in most laboratories. To process the 4D data into a size and format that is more manageable, we developed the
Fig. 2. Basic hardware setup for performing $4 \mathrm{D}$ data acquisition. The video signal from a video camera mounted on the microscope is enhanced by an in-line image processor that applies contrast enhancement, background subtraction, and a degree of frame averaging to reduce video noise. The processed signal is then digitized by a framegrabber card installed in a Macintosh PowerPC computer controlled by our 4D Acquisition software. The acquisition software also controls the focus of the microscope and illumination shutter by means of commands sent from the serial port of the computer to a stage control unit.
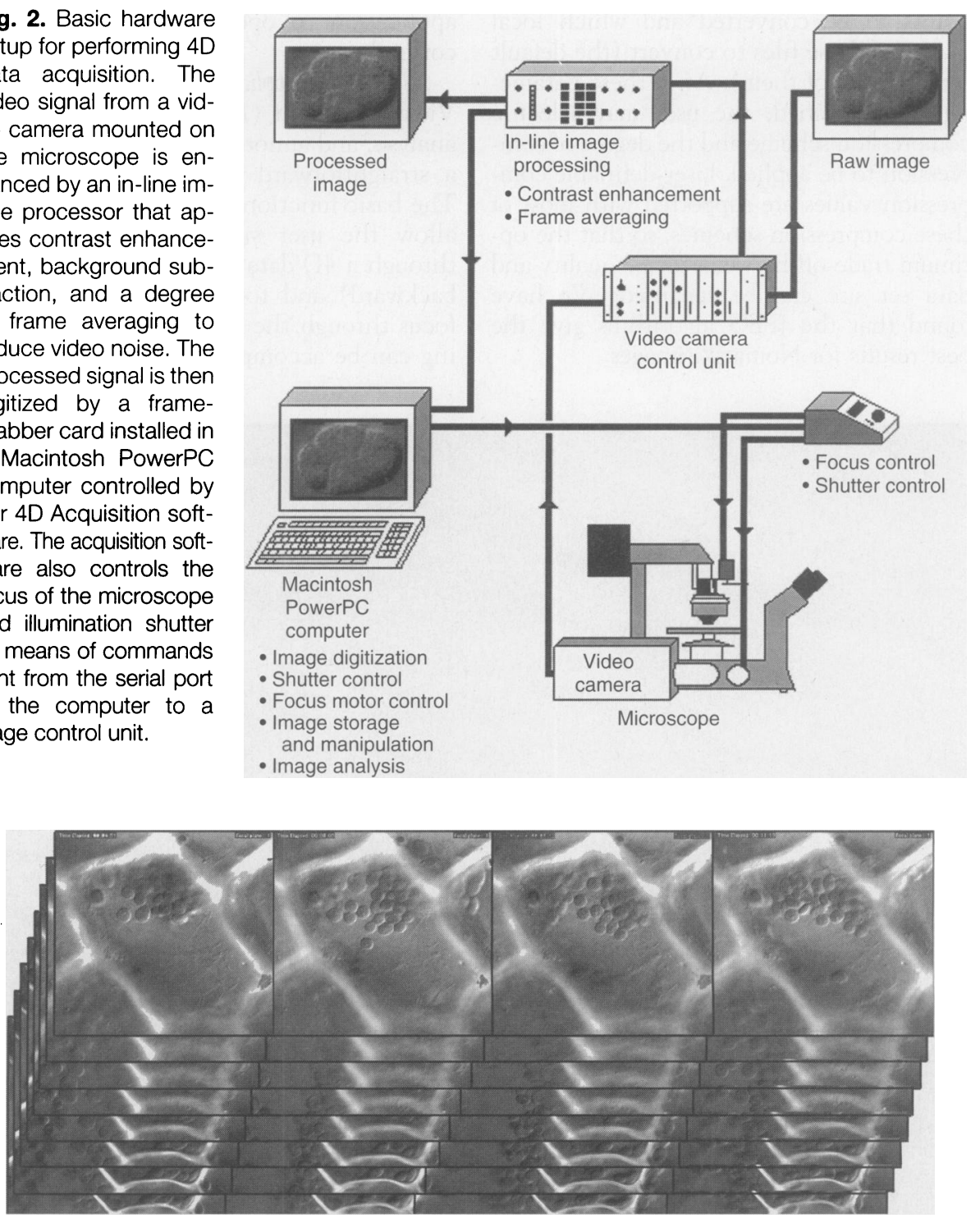

Fig. 3. A typical raw data set of Nomarski images resulting from a 4D data acquisition run. The images show chloroplast movements inside cells from the aquatic plant Elodea. The raw data set consists of a series of 3D stacks, each one containing multiple slices from the top to the bottom of the sample. The 4D Acquisition software controls the hardware shown in Fig. 2 to allow this type of data collection. 
4D Turnaround program. The 4D Turnaround application (14) takes raw 2D or 3D time points and converts them to a Quicktime movie or set of movies.

Quicktime is a system extension (Apple Computer, Inc.) that allows sequences of images to be viewed as a movie (a series of animated frames) on the computer screen. The Quicktime format has become a de facto standard for displaying digital movies and is available for most of the common computer platforms. Quicktime also supports a number of built-in and add-on image compression schemes, including JPEG.

Upon launching the 4D Turnaround application, the user is prompted to open the first time point of the 4D data set. The software recognizes files in TIFF, PICT, PICS, and Bio-Rad's PIC image formats. After opening the first stack of images, the user is prompted for the number of time points to be converted and which focal planes of these files to convert (the default setting is all of them). After these parameters are specified, the user may select a compression scheme and the degree of compression to be applied. User-definable compression values are supported with most of these compression schemes, so that the optimum trade-off between image quality and data set size can be specified. We have found that the JPEG algorithms give the best results for Nomarski images.
When the compression scheme is specified, the Turnaround program creates a separate Quicktime movie file for each focal plane in the first time point, compresses the image information from each focal plane and places it into its respective movie. The program processes the remaining time points in a similar manner, appending the data from each focal plane to the end of its respective movie. The result is a set of Quicktime movies where each movie consists of the data describing the cellular changes from the beginning to the end of the acquisition period at a given focal plane. The movies can also be linked into a single large movie for smoother playback. When all time points have been compressed and added to their respective movies, a "4D format file" is created. The format file contains information about the 4D data set and allows the 4D Viewer application to open processed data sets correctly.

$4 D$ viewer application software. The 4D Viewer program (14) allows the viewing, analysis, and annotation of a 4D data set in a straightforward and intuitive manner. The basic function of the 4D Viewer is to allow the user simultaneously to roam through a 4D data set in time (forward or backward) and to move up or down in focus through the sample (Fig. 4). Roaming can be accomplished either by mouse or keyboard commands and can be done at either the specified frame rate or one frame at a time. A slider on the image window allows quick access to distant time points, and a "go to" function allows the user to rapidly jump to any time point and focal plane in the data set.

When moving through a volume of thousands of images, it is frequently useful to place a few "bookmarks" to provide instant access to specific points of interest. The 4D Viewer allows the user to create bookmarks of this nature and to give them descriptive names. Bookmarks can be saved to and loaded from the computer disk for subsequent viewings of the data set.

Perhaps the most powerful aspect of the 4D Viewer software is the ability to create color overlays for the individual images in the data set. Tools are provided to create circles, squares, straight lines, freehand lines, text, and arrows, in various sizes and colors; any or all of these can overlay the microscope images as they are viewed by the user (Fig. 1). Features of interest in sequential images in the data set can thus be highlighted by dynamic color overlays that will follow the features as they change over time. Additionally, highlighted features can have hidden text (for detailed feature description) associated with them This text can be revealed by clicking on the feature (Fig. 1) and can also be access-

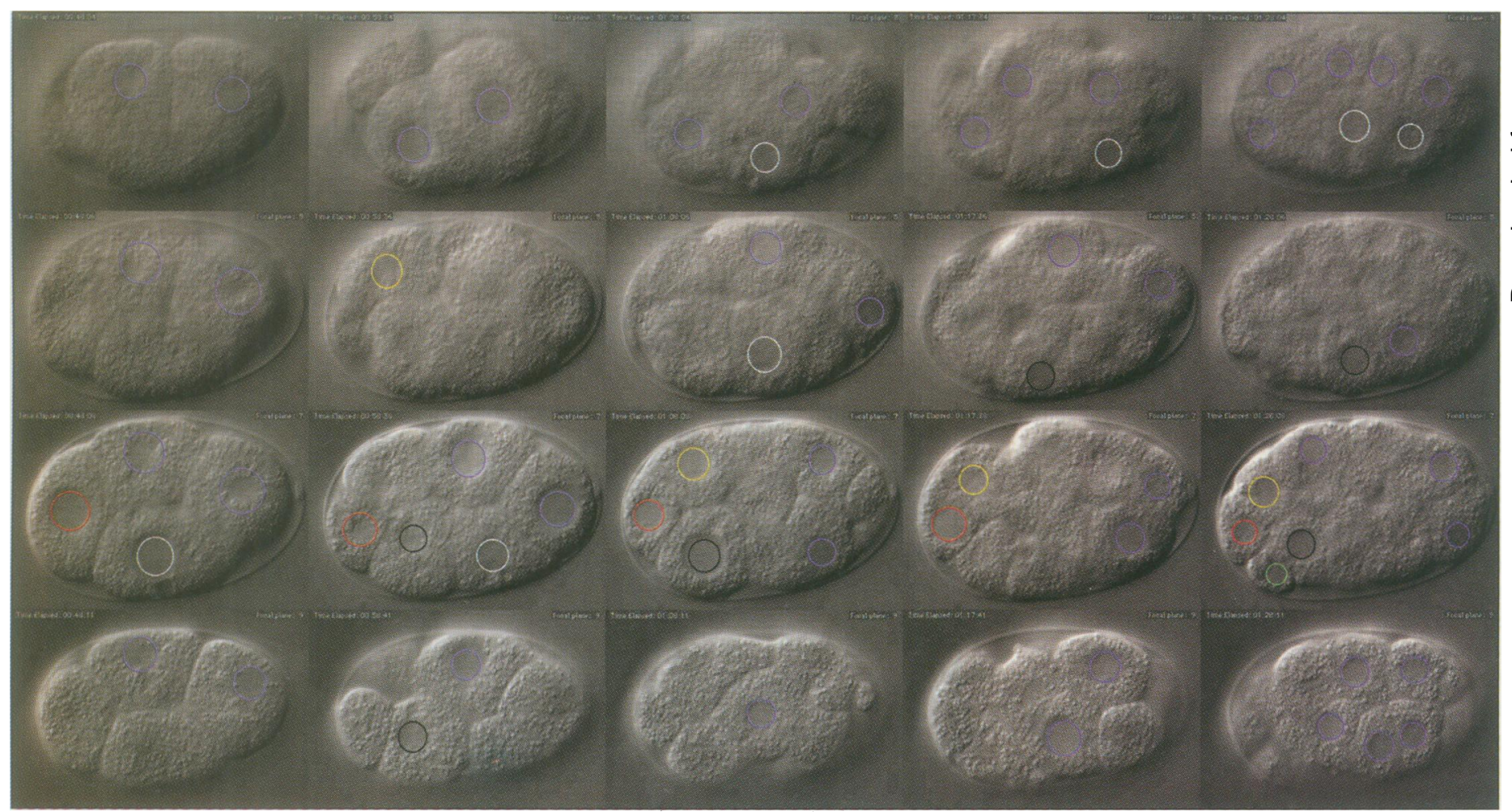

Fig. 4. A sequence of images showing an application of the $4 D$ Viewer software to study $C$. elegans cell lineage. Left to right: changes in elapsed experiment time as the embryo progresses through a period in its early development.
Top to bottom: changes in focal depth from the top to the bottom of the embryo. The nuclei of the cells that are visible in each focal plane have been labeled with colors corresponding to their blastomere identity (see Fig. 5). 


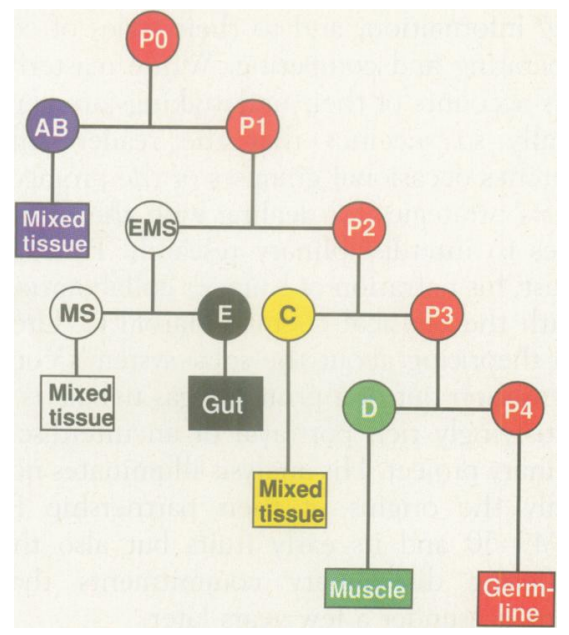

Fig. 5. The embryonic cell lineages of $C$. elegans follow a stereotyped pattern (15). The early divisions generate a set of blastomeres that give rise to all of the tissues of the mature animal. Some, designated $E, D$, and $P 4(15)$, are clonal progenitors of specific tissue types. The colors representing the blastomeres have been used to label the nuclei visible in the optical sections shown in Fig. 4.

ed by a computer search.

As an illustration of the utility of overlays, consider the nucleus of a specific cell of interest. The nucleus can be marked with a red circle in each of the frames of the data set in which it appears, so that as the frames are animated, the red circle will move with the nucleus to show its current position. Also, text that describes the nucleus in more detail can be associated with the red circle in selected frames. At one time point, this text might say "Nucleus 4B, second cell division"; at another, the text might say "Nucleus 4B, entering third cell division." A text search on " $4 \mathrm{~B}$ " would bring up the first instance of the nucleus appearing in the data set, and use of the "find next" command would bring up each subsequent mention of this nucleus.

These overlaid dynamic annotations are not made a permanent component of the original image; they may be added, deleted, or edited at any time by means of a set of tools similar to those available in computer drawing programs. Other applicationspecific tools facilitate the transfer of labels to sequential images. Overlays are stored as objects in a separate disk file distinct from the image data. The use of object coding rather than bit-mapped coding means that overlays take up very little storage space.

\section{Applications of the 4D Image Archival System}

The system was originally developed as an aid for determining cell lineages in C. elegans embryos. The cell lineage of the wild-type organism was determined by direct observation through the microscope (15) before 4D image archiving systems were available. The complete determination of the cell lineage was a major undertaking that took several years to complete. The task was only possible because the lineage is essentially invariant and could be determined piecemeal from many individuals. The 4D image archival system should now allow the determination of many of the embryonic lineages in an individual embryo to be done in a matter of days (Figs. 4 and 5). The 4D technology makes it feasible to determine cell lineages in situations where there-is variability, such as in embryonic mutants, or in individual animals that have undergone laser surgery.

The 4D system is being increasingly used to study intracellular movements. A 4D analysis enabled the pattern of cytoplasmic streaming within a cell to be determined (7). Current areas of study at IMR include mechanisms of mitochondrial and chloroplast movements in the aquatic plant Elodea (Fig. 3), pigment granule movements in stimulated keratocytes, and contraction of smooth muscle cells in response to specific stimuli.

In addition to its use as a research tool, the system has considerable potential as an educational aid. For example, a complete annotated 4D data set of the embryonic development of C. elegans could be produced. This data set, together with the viewer program, could be distributed as a CD-ROM or set up on the Internet. With these data, a student would be able to identify all the cells in the developing embryo by means of overlays and associated text notes. He or she would then be able to follow the fates of the identified cells through the major developmental events, such as gastrulation and morphogenesis. The 4D imaging system that we have described is freely available and can be downloaded from the Internet (14).

\section{REFERENCES AND NOTES}

1. M. Chalfie, Photochem. Photobiol. 62, 651 (1995).

2. J. G. White, W. B. Amos, M. Fordham, J. Cell Biol. 105, 41 (1987).

3. W. Denk, J. H. Strickler, W. W. Webb, Science $\mathbf{2 4 8}$ 73 (1990)

4. D. A. Agard, Y. Hiraoka, P. Shaw, J. W. Sedat, Meth ods Cell Biol. 30, 353 (1989).

5. J. S. Minden, D. A. Agard, J. W. Sedat, B. M. Alberts, J. Cell Biol. 109, 505 (1989).

6. R. A. Driebin, L. Carpenter, P. Hanranhan, Computer Graphics 22, 65 (1988).

7. S. N. Hird and J. G. White, J. Cell Biol. 121, 1343 (1993).

8. S. Inoue and T. D. Inoue, Biol. Bull. 187, 232 (1994).

9. R. Schnabel, Mech. Dev. 34, 85 (1991).

10. A. Fire [Comput. Appl. Biosci. 10, 443 (1994)] describes a $4 \mathrm{D}$ system implemented on an IBM PC- compatible computer.

11. The $4 D$ live cell imaging workstation at IMR uses the following hardware; however, many combinations of hardware may be used for 4D imaging, and this list is neither a recommendation of what to buy nor an indication of what is necessary to do this type of data collection. Microscope: Nikon Diaphot 200 with Nomarski optics and a Nikon oil immersion lens (100X, numerical aperture 1.4). Video camera: Sierra Scientific Video Standard LSV-1 Vidicon with Nikon zoom lens $(0.75 \times$ to $2.5 \times)$. Image processor: Dage DSP2000. Frame grabber: Scion LG 3. Focus drive: ASI model 85. Shutter drive: ASI SC-2 shutter controller (the Ludl MAC 1000 and MAC 2000 systems are also supported). Computer: Macintosh Quadra 950, PPC upgrade card, 25 megabytes of RAM, 1-gigabyte internal hard drive, 1.2-gigabyte APS magnetooptical drive (for archival purposes). Recently, a second system has been set up with a Macintosh 7600/ 120 computer and a Scion AG 5 frame grabber. The AG 5 frame grabber can perform frame averaging, thereby obviatina the need for a separate image processor.

12. The $4 \mathrm{D}$ Acquisition software is based on $\mathrm{NIH}$ Image a popular shareware image processing program and is basically an extensive set of control macros for $\mathrm{NIH}$ Image that allows the user to configure and control the image acquisition conditions through a series of graphic user interfaces. The $\mathrm{NIH}$ Image program is a freely available general-purpose image processing system for Macintosh computers. It features a comprehensive macro language that was used to implement the data capture commands of the system. A user can easily extend or customize his or her acquisition software by editing and modifying the macros. NIH Image was written and is maintained by W. Rasband. Executable code and associated documentation can be obtained from the $\mathrm{NIH}$ Image World Wide Web site, http://rsb.info.nih. gov/nih-image/

13. We found that we could obtain up to $25 \%$ more data compression and better final image quality if we filtered out residual electronic or photon noise from the images. For Nomarski imaging, we use a four-frame running-average filter implemented on a Dage DSP 2000 image processor. On another system, we use a four-frame average on a Scion AG 5 integrating frame grabber, thereby obviating the need for a separate image processor.

14. IMR's 4D Acquisition software, 4D Turnaround program, 4D Viewer program, and the related documentation are available by anonymous FTP from: Address: ftp2. bocklabs. wisc.edu

User name: anonymous

Password: your e-mail address

Directory: pub/lmb/imr/4D_Viewer

Folders: "Acquisition Macros," "Turnaround," and "Viewer," respectively

Alternatively, the software can be downloaded from IMR's Web site, http://www.bocklabs.wisc.edu/ $\mathrm{imr} / \mathrm{imr}$.html. This site also contains further information on 4D microscopy, including equipment purchase considerations, vendor addresses, and equipment costs. If you wish to be put on a list to receive information about future upgrades or releases of IMR's 4D software, send an e-mail outlining your request to cfthoma1@facstaff.wisc.edu with your name, mailing address, and phone number. Note that turning around large data sets can be time-consuming. On a Macintosh 8500 , the $4 D$ Turnaround program will process 10 time points consisting of 20 focal planes of images of 300 by 400 pixels in $\sim 1.5 \mathrm{~min}$.

15. J. E. Sulston, E. Schierenberg, J. G. White, J. N Thomson, Dev. Biol. 78, 64 (1983)

16. We thank V. Centonze for ongoing support and discussions, V. Centonze and C. Lavin for constructive comments on the manuscript, D. Wokosin for setting up the instrumentation, W. Russin for the Elodea sample shown in Fig. 3, and the users of the system for their suggestions for improvements and identification of bugs. Supported by NIH grant P41-RR00570-26. 